\title{
Die Kritik der gesellschaftlichen Verhältnisse in Ma- rek Fiedors Bühnenbearbeitung von Franz Kafkas Der Proceß für das Teatr Polski in Poznań 2004
}

Franz Kafka należy do tych niezwykłych autorów, którzy nigdy nie napisawszy żadnego dramatu, na trwałe utorowali sobie drogę na sceny teatralne. Nie inaczej rzecz przedstawia się w Polsce, gdzie wielu reżyserów teatralnych sięga po opowiadania i powieści Kafki, aby zaprezentować ich wersję sceniczną. Gdy zadania takiego podejmuje się jeden z najzdolniejszych polskich reżyserów, jak dzieje się to w przypadku Marka Fiedora, rzecz wymaga szczególnej uwagi. Niniejszy artykuł podejmuje próbę analizy adaptacji scenicznej Procesu Kafki w ujęciu Marka Fiedora z 2004 roku i wskazuje na to, iż mimo sceptycznego podejścia wielu naukowców i znawców teatru teksty Kafki z sukcesem można przenieść na scenę.

Franz Kafka gehört zu den außergewöhnlichen Autoren, die nie ein Drama geschrieben haben und doch immer wieder auf den Theaterbühnen gespielt werden. Auch in Polen greifen sehr oft Theaterregisseure auf Kafkas Romane und Erzählungen zurück, um sie für das Theater zu adaptieren. Wenn dies - wie es bei Marek Fiedor der Fall ist - einer der erfolgreichsten polnischen Regisseure tut, so erfordert diese Tatsache besondere Aufmerksamkeit. Der folgende Beitrag hat Fiedors Bühnenbearbeitung von Kafkas Der Proce $\beta$ aus dem Jahre 2004 zum Gegenstand seiner Analyse und zeigt, dass Kafkas Texte sich trotz der großen Skepsis vieler Wissenschaftler erfolgreich auf die Bühne bringen lassen.

Franz Kafka is one of those exceptional authors who never wrote a drama and despite this paved his way to theatrical stages. The situation is not different in Poland where numerous theatre directors reach for short stories and novels of Kafka in order to present their scenic version. When one of the most talented Polish directors embarks on such a task, as it is in the case with Marek Fiedor, this requires special attention. The article makes an attempt at the analysis of stage adaptation of The Trial by Kafka based on Marek Fiedor's vision dated from 2004 and demonstrates that in spite of 
Eliza Szymańska

sceptical opinions of many scholars and theatre experts, the texts of Kafka can be successfully transferred on the stage.

\section{Vorbemerkung}

Dieser Beitrag folgt der gegenwärtig immer öfter angestrebten Tendenz, literatur- und theaterwissenschaftliche Untersuchungsperspektiven miteinander zu verbinden (BAYERDÖRFER 2007). Als Ausgangspunkt der Überlegungen soll Andrzej Żurowskis Artikel Kafka im polnischen Theater. Erforschung eines Untersuchungsthemas dienen (ŻUROWSKI 2005). Żurowski gibt offen zu, dass das gegenwärtige Theater nicht immer seinem Geschmack entspricht. Trotzdem schreibt er weiter: „Mit Bescheidenheit schaue ich den gegenwärtigen Aufführungen zu, glücklich darüber, dass sie - auch dank Kafka - die Art des Denkens und Fühlens der Menschen aus der Generation meiner Tochter und meines Sohnes wiedergeben." (ŻUROWSKI 2005:245) Diese Worte können m.E. gut auf Marek Fiedors Bühnenadaption von Franz Kafkas Der Proce $\beta$ bezogen werden, denn es handelt sich um eine moderne, der Ästhetik des gegenwärtigen Theaters entsprechende Aufführung.

\section{Die Entstehungsgeschichte}

Marek Fiedor beschloss aus zwei Gründen, eine Bühnenbearbeitung von Der Proce $\beta$ zu präsentieren. Zum einen wollte er einen neuen Interpretationsschlüssel zu einem Roman finden, der bis jetzt vorwiegend als Geschichte über die Versklavung des Individuums durch ein totalitäres System aufgefasst wird. Zum anderen wollte Fiedor mit dem in Polen geltenden Stereotyp brechen, das den Prager Dichter mit der Hauptgestalt seines Romans identifizierte. Nach Fiedors Auffassung ist Josef K. nicht ein kleines Rad im bürokratischen Uhrwerk, sondern ein Repräsentant des Establishments und damit auch Teil von dessen Antriebskraft. Fiedor sieht die Arbeit im Theater als Interpretation der Wirklichkeit und nicht der Literatur. Das erklärt zum größten Teil seinen sehr lockeren Umgang mit dem Originaltext. Zugleich erkennt man darin die Faszination Fiedors für die Arbeit von Regisseuren wie Jerzy Jarocki, Jerzy Grzegorzewski und Krystian Lupa.

Fiedors Anliegen ist nicht eine Nachahmung von Kafkas Welt auf der Bühne, er will auch die Romanwirklichkeit nicht originalgetreu abbilden. Der Regisseur kreiert eine ganz neue, eigene Welt. Dabei stützt er sich nicht nur auf den Romantext (einschließlich der Teile, die nicht in die Brod-Edition einge- 
gangen sind), sondern auch auf die Tagebücher, Briefe oder Erzählungen Kafkas. Er meidet aber vorsätzlich alle Interpretationen von Kafkas Schaffen, da er den Dichter ,aus erster Hand“ (GUDERIAN-CZAPLIŃSKA 2004:35) kennenlernen möchte. Einzige zusätzliche Quelle ist für Fiedor die Biographie Kafkas, da sie ihm hilfreich zu sein scheint, um die von Kafka dargebotene Vision der Wirklichkeit zu verstehen. Der Regisseur sieht die Welt Kafkas als eine Welt, in der die immer aktuelle Vorahnung einer Bedrohung dominiert. Der Prozess Josef K.s bietet eine einzigartige Chance, die hermetische Welt des Erfolgs, in der der Protagonist lebt, zu verlassen und Kontakt mit einem anderen, einfachen Menschen anzuknüpfen. Diese Möglichkeit will der Held aber nicht wirklich ergreifen. Fiedor sieht Kafka als einen Realisten, der aber zugleich metaphysisch auszulegen wäre. Diese Art und Weise, Kafkas Werk aufzufassen, ist in der Bühnenbearbeitung deutlich zu sehen. Die ersten Szenen, gespielt im Geiste des Realismus, machen im Laufe des Bühnengeschehens immer deutlicher Szenen mit metaphysischem Charakter Platz.

\section{Struktur- und Inhaltsanalyse}

Die Bühnenbearbeitung von Der Proce $\beta^{1}$ durch Marek Fiedor besteht aus 19 Szenen, deren Aufbau sich nur locker an die Romankonstruktion anlehnt, ohne deren Chronologie zu bewahren. Der Regisseur fügte zahlreiche Szenen ein, die keine Entsprechung im Roman haben. Ihre Funktion besteht entweder in der Aktualisierung des Bühnengeschehens (Sonntag, Happening, Demonstration) oder in der Vertiefung des psychologischen Porträts des Protagonisten (Lifestyle, Geburtstag).

In der ersten Szene (Der Aufruf), die sich an das neunte Kapitel des Romans (Im Dom) anlehnt, wird Josef K., der sich im Dom befindet, vom Priester ermahnt, sich Gedanken über sein bisheriges Leben zu machen. Die bereits in dem Motto zum Stück erwähnte Unmöglichkeit der Trennung zwischen der äußeren und der inneren Welt des Protagonisten - es handelt sich um die Worte Elias Canettis aus dem Essay Der andere Prozeß. Kafkas Briefe an Felice (CANETTI 2005) - wird in dieser Szene deutlich. Die äußere Welt Josef

1 Der Proceß nach Franz Kafka. Bühnenbearbeitung Marek Fiedors. Teatr Polski, Poznań 2004 [Theaterexemplar]. Im Folgenden unter der Sigle $P F$ zitiert. Alle Übersetzungen aus der Textfassung der Bühnenadaption Fiedors stammen von der Autorin dieses Beitrags. 
K.s - darüber geben uns die folgenden Szenen des Stückes Auskunft - ist die Welt des Erfolgs. Seine innere Welt dagegen ist öde und leer. Josef K. macht sich keine Gedanken über seine geistige Entwicklung. Da er ständig in Sorge um die eigene berufliche Karriere ist, fehlt ihm die Zeit dazu. Da er meint, dass die Trennung der beiden Welten möglich ist, versucht der Priester, ihn vom Gegenteil zu überzeugen. Der Prozess kann als ein Versuch verstanden werden, Josef K. bewusstzumachen, dass ein Leben, das nur auf materiellen Werten basiert, mit dem Tode der inneren Welt einhergeht, was nur den körperlichen Tod als Folge haben kann.

In der zweiten Szene (Verhaftung) wird Josef K. verhaftet. Aber Marek Fiedor wechselt die Perspektive und lässt uns die Verhaftung mit den Augen von K.s Nachbarn (der Alte, die Alte und der Sohn) verfolgen. Die Verhältnisse in dieser Familie ähneln sehr denjenigen, die in der Familie Kafka herrschten. Der autoritäre, etwas robuste Vater, die untergeordnete, milde Ehefrau und der verzweifelte Sohn. Am Frühstückstisch sitzend, kommentieren sie die Geschehnisse in K.s Wohnung, die sie neugierig durch das Fenster beobachten. Der dadurch bewirkte Perspektivenwechsel ist ein Signal, dass der Prozess sich fortwährend in der Öffentlichkeit abspielen wird. Marek Fiedor hatte am Anfang der Arbeit mit dem Roman Kafkas die Idee, den Proce $\beta$ als eine Geschichte über das Beobachtet-Werden zu präsentieren, was nach Ansicht EWA GUDERIAN-CZAPLIŃSKAs (2004) heutzutage höchst aktuell sei. Die Idee wurde aufgegeben, aber ihre Reminiszenzen fanden in die zweite Szene Eingang. Es gibt in dieser Szene noch weitere Beobachter - zwei Engel, die von da an immer wieder in den Schlüsselmomenten der Bühnenbearbeitung erscheinen werden, vor allem dann, wenn Josef K. von seinem Prozess immer mehr eingeengt wird.

In der dritten Szene (Lust) bekommen wir zum ersten Mal Gelegenheit zu hören, was Josef K. selbst zu sagen hat zu dem, was ihm widerfahren ist. In dieser Szene sind die Aktualisierungsverfahren des Regisseurs besonders deutlich zu sehen. Zum einen sprechen die Protagonisten - Josef K. und Fräulein Bürstner - die Sprache junger Menschen. Alle Wörter mit archaischem Charakter werden konsequent übergangen oder ersetzt. Zum anderen wohnt Josef K. nicht in einer Pension (deshalb wird auch auf die Person Frau Grubachs verzichtet), sondern in einer exklusiven Wohnung in einem TrendViertel. Was er sagt, macht ihn dem Zuschauer nicht gerade sympathisch. Seine etwas arrogante, überhebliche Art ist besonders in den Worten zu spüren, die er nach dem Verlassen von Fräuleins Bürstners Wohnung äußert. „Eine Assistentin, die werde ich noch kriegen“ (PF:10), sagt sich K., der da- 
mit Gedanken aus dem vierten Kapitel des Romans wiedergibt. Aus diesem Kapitel werden noch andere wichtige Worte übernommen. Am Anfang des Gesprächs zwischen K. und Fräulein Bürstner äußert sie ihre Verwunderung über die Tatsache, dass er mit ihr sprechen möchte, da er sich normalerweise für die Nachbarn nicht interessiert, was uns an die Worte von Fräulein Montag erinnert. Fiedor beschloss, diese Worte in seinen Proceß zu übernehmen, da sie K.s Einstellung gegenüber seinen Mitmenschen treffend wiedergeben.

K. schenkt seinen Mitmenschen keine Aufmerksamkeit, da er zu sehr auf die eigene Karriere bedacht ist, was in der vierten Szene (Karriere) deutlich zum Vorschein kommt. Wir sehen K. in seinem Element - bei der Arbeit in der Bank. Er hat alles unter Kontrolle, macht mehrere Sachen gleichzeitig, gibt sich höchst professionell. Zugleich ist er jedoch skrupellos gegenüber einem Kunden, der bankrott gegangen ist und der randaliert, weil er sich von der Bank betrogen fühlt. Aus K.s Worten lässt sich schließen, dass er nicht zum ersten Mal mit so etwas konfrontiert ist. Er gibt einem jüngeren Kollegen Ratschläge, wie man in solchen Situationen zu handeln hat. Es wird deutlich, dass K. vollkommen die Regeln einer Welt akzeptiert, in der der Schwächere vom Stärkeren ,aufgefressen“ "wird.

Die fünfte Szene des Stückes zeigt den Lebensstil Josef K.s (Lifestyle). Er kommt nach der Arbeit mit einem Pizzakarton in der Hand nach Hause, zieht sich schnell um. Dann macht er Kraftübungen, isst gleichzeitig seine Pizza und wiederholt italienische Wörter aus dem Bereich des Bankwesens von einer $\mathrm{CD} .{ }^{2} \mathrm{Um}$ etwas zu erreichen, muss K. sich außerhalb der Bürostunden weiterbilden. Dabei hat er keine Zeit, etwas Ordentliches zu essen, geschweige denn, es sich selbst zuzubereiten. Zugleich muss er auch immer in Form sein, denn die Welt des Erfolgs, in der er sich zu Hause fühlt, akzeptiert keine Menschen, die kein musterhaftes Aussehen haben. Man müsste sich fragen, wo hier die Zeit für die Reflexion über das eigene Leben bleibt? Diese Frage stellt sich Josef K. jedoch nicht. Abermals lehnt sich das von K. entworfene Bild eng an die Romanvorlage an. Kafka beschreibt einen jungen

2 Dass K. italienische Wörter lernt, ist kein Zufall. Fiedor spielt hier auf die Tatsache an, dass Josef K. bei Kafka im vorletzten Kapitel einen italienischen Kunden der Bank durch den Dom führen soll. Im Roman lesen wir: „Es war ein sehr regnerischer, stürmischer Morgen, als K. voll Ärger über den Tag, der ihm bevorstand, schon um sieben Uhr ins Bureau kam, um wenigstens einige Arbeit noch fertigzubringen, ehe der Besuch ihn allem entziehen würde. Er war sehr müde, denn er hatte die halbe Nacht mit dem Studium einer italienischen Grammatik verbracht, um sich ein wenig vorzubereiten [...].“ (KAFKA 1997:533) 
Karrieremenschen in den Realien, die seinen Zeiten entsprachen. Fiedor ,übersetzt ${ }^{\star}$ das Bild in die heutige Zeit, ebenso in der nächsten Szene, wenn er K. nicht in einer Bierstube (wo Josef K. aus dem Roman seine Abende verbrachte), sondern in einer - wie es sich für die finanziellen Eliten gehört der angesagtesten Discos der Stadt seinen Geburtstag feiern lässt. Bevor K. erscheint, hören wir dem Gespräch seiner Kollegen zu. Der eine erzählt, unter den eifersüchtigen Blicken der zwei anderen, von der neugekauften Wohnung. Wir bekommen also auch direkt die Antwort auf die Frage, wohin dieser Wettbewerb führen soll. Es wird ein konkretes Ziel verfolgt: möglichst viel an materiellen Gütern zu sammeln, um damit protzen zu können.

Während der Unterhaltung wird K. auf seinem Handy angerufen. An seinen Antworten merken wir, dass es sich um den Termin der ersten Untersuchung handelt. Darauf weisen aber auch die Gestalten der beiden Engel hin, die im Hintergrund erscheinen. Josef $\mathrm{K}$. spricht mit seinem Gesprächspartner auf eine überhebliche, grobe Art. Man merkt, dass er, der daran gewöhnt ist, Macht zu haben, nur ungern den Befehlen anderer folgt. Dass er trotzdem zur ersten Untersuchung geht, obwohl er im Gespräch beteuert, keinen Grund für diesen Termin zu sehen, macht deutlich, dass K. tief in seinem Inneren fühlt, dass es seine Pflicht sei, vor Gericht zu erscheinen.

Die siebte Szene (Sonntag) ist eine Art Einführung in die Untersuchungsszene. K. bekommt während eines Spaziergangs Einblick in das Leben, dass er, eingesperrt zwischen seiner Bank und seinem luxuriösen Wohnviertel, an sich nicht kennt. Es sind ein paar simultane Szenen: Zeugen Jehovas versuchen, ein vorübergehendes Paar über die heilende Kraft der Liebe zu belehren in einer Gesellschaft, für die nur Geld, Macht und Karriere zählen. Ein verlobtes Paar streitet miteinander. Zwei ältere Passanten sind empört, dass zwei Automechaniker am Sonntag arbeiten. Und eben diese Mechaniker erzählen sich makabre Geschichten, unter anderem über eine junge Frau, die aus Verzweiflung, Armut und Hunger ihr neunmonatiges Baby erdrosselt hat. Das ist eine Welt, die K. nicht kennt, für die er aber seiner konformistischen Einstellung wegen mitverantwortlich ist. Dafür steht die Tatsache, dass diese Menschen in der nächsten Szene der ersten Untersuchung beiwohnen. Sie sind K.s Ankläger. Unter ihnen sieht man auch den Priester - K.s Gewissen, das er durch seine Rede überspielen will. Anhand der Rede wird klar, dass K. versucht, alle von seiner Unschuld zu überzeugen. Er wird aber vom Regisseur schuldig gesprochen - unter anderem des Versagens im Umgang mit anderen Menschen, was in der neunten Szene (Falle) deutlich wird. 
K. befindet sich wieder in der Bank und führt ein Einstellungsgespräch mit einem Bewerber um die Stelle des Dieners. Die Klagen des Mannes über seine schwere Situation (er war lange Zeit krank und ist deswegen arbeitslos, hat vier Kinder und sucht verzweifelt eine Stelle) machen auf K. einen sehr schlechten Eindruck. Seine Äußerung, „Wir sind keine Wohltätigkeitsorganisation“ ( $P F: 22)$, zeigt K. als einen rücksichtslosen Menschen, dem jedes Mitgefühl für seine Mitmenschen fehlt. Diese Worte scheinen im Widerspruch zur Aussage der im Hintergrund erscheinenden Mutter K.s zu stehen:

\section{MUTTER}

Josef hatte nie die Gabe, wie die anderen Menschen, seine Liebe zu zeigen. Ich weiß, dass er mich innig liebt, trotzdem hat er weder mir noch seinem Vater seine Liebe gezeigt. Vielleicht ist er für das Heiraten nicht geschaffen, da er ausschließlich an seine Arbeit denkt, die für ihn am wichtigsten im Leben ist. Letztendlich ist er ein Direktor... oder fast ein Direktor. Er hat's doch zu etwas gebracht. Er ist sensibel und schwach, das ist aber nicht die Art von Schwäche, über die man beschämt sein müsste. (PF:23)

Die Mutter bezeugt zwar, dass ihr Sohn gefühllos sei, im Grunde genommen hält sie ihn jedoch für sensibel und schwach. Was zunächst wie ein Witz klingt, wenn wir bedenken, was wir gesehen haben, aber durchaus der Wahrheit entspricht. Wäre K. nicht sensibel und schwach, wäre es nie zu jenem Prozess gekommen, den man als Ausbruch von K.s Gewissensbissen verstehen kann. K. fühlt sich vorerst noch unbewusst schuldig, was davon zeugt, dass er tief im Inneren das Gefühl hat, auf menschlicher Ebene versagt zu haben. Das bezeugt auch das Gespräch mit dem Onkel in der nächsten Szene (Der Onkel). Der Onkel macht K. schwerste Vorwürfe, als er erfährt, dass er seine Mutter seit zwei Jahren nicht mehr besucht hat. In der Vernachlässigung der Familie liegt, nach der Meinung des Onkels, K.s Hauptschuld.

Auf dem Weg zum Advokaten in der zehnten Szene (Happening) trifft K. auf eine Gruppe von Globalisierungsgegnern, also Menschen, die gegen eine Welt protestieren, deren beispielhafter Repräsentant K. ist. Fiedor zeigt Demonstranten, die gegen die Ausbeutung der Menschen durch Konzerne protestieren. Es stellt sich die Frage, ob K. sich diesen Menschen nicht anschließen sollte, um so seine Schuld begleichen zu können. Dazu ist er allerdings nicht bereit, was in der nächsten Szene deutlich wird (Gehorsam). Die Gespräche dieser Szene stützen sich weitgehend auf das, was wir im Roman lesen können. Mit einer kleinen, aber bedeutenden Ausnahme. Im Roman versucht Leni, K. dazu zu überreden, dass er vor Gericht ein Geständnis ablegt. Im Stück verlangt sie von ihm, dass er sich für schuldig erklärt, denn erst dann könne sie ihm helfen. Das bezeugt, dass K.s Prozess sich in seinem 
Eliza Szymańska

Gewissen abspielt und dass die Ankläger Menschen aus seiner Umgebung sind. Erst das Eingeständnis vor sich selbst, dass dies ein falsches Leben ist, bietet den Ausgangspunkt einer Besserung. K. ist dazu aber nicht bereit, und in seinen Gesten wird deutlich, dass er auch die Frau instrumentell behandelt und zu eigenen Zielen ausnutzen will. In der nächsten Szene (Sekte) wird K.s Verhalten vom Onkel kritisiert. Im Hintergrund singt eine Gruppe von Krishna-Anhängern. K. wird somit ein zu seinem eigenen öden Leben alternativer Lebensstil präsentiert.

Am Anfang der dreizehnten Szene (Krise) treffen wir K. erneut in der Bank. Es wird deutlich, dass der Prozess Josef K. dermaßen in Anspruch genommen hat, dass er jetzt keine Zeit mehr für seine Kunden hat. In die eigenen Gedanken versunken, sitzt er bis spät in den Abend. K. beginnt ein Gespräch mit einer Putzfrau, aus dem hervorgeht, dass er sich dessen bewusst ist, dass er sein bisheriges Leben einer eingehenden Analyse unterziehen sollte. Er hat jedoch nicht vor, dies zu tun, denn: ,[...] damit man sich selber verteidigen kann, muss man alles andere lassen... Und die Arbeit? Und die Karriere?“ $(P F: 34)$ K. erläutert der Frau auch seine Lebenstaktik. Er will zu allen nett sein, damit sie ihn mögen. Dann kann er nämlich ohne Umstände hinterhältig handeln. Das Gespräch mit der Putzfrau dreht sich vor allem um das Gericht und dessen Repräsentanten. Es ist bedeutend, dass wir diese im Stück auf der Bühne niemals sehen, sondern von ihnen nur ab und zu hören. Das Gericht wird dadurch höchst enigmatisch. Es entsteht der Eindruck, dass man das Gericht nie erreichen kann, was der Darstellungsweise dieser Institution im Roman entspricht. Die meisten Informationen über das Gericht bekommt K. bei Titorelli. Bevor er zu ihm gelangt, trifft er in der vierzehnten Szene (Demonstration) Arbeiter, die für die Verbesserung ihrer Situation demonstrieren. Ihr Sprechchor „Diebe, Diebe“ $(P F: 35)$ ist ein Angriff auch auf K., da er die Welt der großen Finanzen repräsentiert.

Die Szene beim Maler Titorelli (Stickige Luft) bildet eine Art Zäsur in Fiedors Bühnenbearbeitung. Von diesem Moment an verzichtet der Regisseur auf die Aktualisierung des Bühnengeschehens und lehnt sich an die Romanvorlage an. Dadurch wird der metaphysische Charakter des Proce $\beta$ deutlich, den bis jetzt nur die Anwesenheit der Engel angedeutet hat. Wir haben es in dieser Szene mit einem Bruch in der vorherigen, realistischen Darstellung der Handlung zu tun, was Fiedors Vorhaben entspricht, dem Zuschauer verschiedene Interpretationsmöglichkeiten anzubieten (GUDERIAN-CZAPLIŃSKA 2004). Plötzlich wird der Prozess Josef K.s als ein Ereignis gezeigt, das jedem widerfahren könnte. Josef $K$. wird ein Jedermann. Die Zäsur wird auch 
äußerlich markant. Die modernen Möbel machen alten Schränken, Kommoden und Sofas Platz. Außerdem wird die Atmosphäre einer Traumwirklichkeit hervorgerufen, wenn K. bei dem Versuch, das Atelier des Malers zu verlassen, direkt in den Dom spaziert. Ihren Höhepunkt erreicht diese Atmosphäre in der nächsten Szene (Kanzleien), wenn K. von dem Maler durch Gerichtskanzleien geführt wird. In dieser und in der nächsten Szene (Verachtung) wird deutlich, dass K. nur darauf bedacht ist, den Prozess möglichst weit von sich wegzuschieben. In der vorletzten Szene treffen wir Josef K. erneut im Dom. Dadurch wird zwischen der ersten und achtzehnten Szene des Stückes eine Klammer geschaffen. Die Handlung beschreibt einen Kreis, und wir treffen K. wieder am Ausgangspunkt. Der einjährige Prozess hat ihn nichts gelehrt, was in dem Gespräch mit dem Priester deutlich wird. Aber durch den Priester erfährt K., was sein Prozess eigentlich bedeutet. Im Bewusstsein dessen wartet er in der neunzehnten Szene ruhig auf die Henker. Der Titel der Szene (Scham) bezieht sich auf K.s Gefühle, die er hegt, weil er die Lüge seines Lebens nicht rechtzeitig bemerkt hat. Die Lüge, dass seine Lebensweise ohne Einfluss darauf sei, was für ein Mensch er ist. Die Lüge, dass in der Welt des Establishments nur Grausamkeit und Rücksichtslosigkeit als die einzigen Verhaltensweisen möglich seien.

\section{Szenische Gestaltungsmittel}

Das Bühnenbild für Fiedors Bühnenbearbeitung von Der Proceß ist das Werk von Jan Kozikowski. Als Hauptelemente dienen hohe Glaswände, die eine monumentale, großstädtische Architektur nachahmen. Das Interieur ist sehr modern und asketisch. Es knüpft an die Lebensweise K.s an, der ein modernes, aber im Kontakt mit anderen Menschen asketisches Leben führt. Eine Metallkonstruktion in Form eines Würfels erfüllt verschiedene Funktionen. Es ist das Interieur eines modernen Bürohauses. In den Szenen in den Gerichtskanzleien ähnelt es einer Falle, in der K. gefangen ist. Es dient auch als Übergang zwischen der realen, heutigen Welt und der metaphysischen, unverständlichen Welt des Prozesses. Die irreale Atmosphäre steigern noch überall aus den Kulissen herausragende Elemente, vor allem Büromöbel. Sämtliche Bühnenbildelemente werden in der Finalszene beseitigt. K. befindet sich jetzt auf der leeren Bühne, allein mit seinem Urteil, und spricht den Bericht über seinen eigenen Tod in ein von der Decke hängendes Mikrofon. Seine anfangs ruhige Stimme steigert sich mit der Zeit bis in ein Schreien. Die Wirkung der Sprache wird meisterhaft vom Lichtspiel ergänzt, 
Eliza Szymańska

für dessen Regie Grzegorz Cwalina verantwortlich war. Das Licht wird so geführt, dass manche Wörter länger in der Erinnerung bleiben, da sie sehr suggestiv wirken. Das Licht unterstreicht auch überzeugend die Atmosphäre, die wir aus Kafkas Roman kennen - zum Beispiel, wenn wir uns in den Gerichtskanzleien befinden und das verdünnte weißlich-trübe Licht die stickige Luft und den schwebenden Staub geradezu spürbar macht. Die in der Szene mit Huld herrschende Dämmerung lässt an die Altersschwäche des Advokaten denken. Genauso wie das alte, kurze Bett, in dem der Advokat, nur mit einem Schlafrock bekleidet, seine Tage verbringt. ${ }^{3}$ Und die schwach beleuchtete erste Szene der Bühnenbearbeitung, in der mit dem Rücken zum Publikum stehende Menschen einen nachklingenden Ton von sich geben, ist eine Ankündigung des beginnenden Prozesses. Die in dieser Szene herrschende Dunkelheit spiegelt die Situation Josef K.s wider, der bei seinem Prozess das Gefühl nicht loswerden kann, im Dunkeln zu tappen. In dem Stück ist auch die Geräuschkulisse sehr suggestiv. Plötzliches Knirschen, dumpfe Geräusche und Stadtlärm stehen für die K. bis jetzt unbekannte Welt, die nach eigenen Gesetzen funktioniert. Dieser Eindruck wird durch die Musik von Tomasz Hynek verstärkt. Die Kostüme bezeichnen klar die Handlungszeit - Der Proce $\beta$ spielt hier und jetzt. Zugleich geben sie uns Auskunft über den sozialen Status der einzelnen Personen. Josef K. in seinem teuren Anzug, mit dem unentbehrlichen Accessoire, seinem Handy, erscheint als Mensch des Erfolgs. Josef K. definiert sich durch das Körperliche. Er ist ständig darum bemüht, dass seine Schritte entschieden wirken, dass es aussieht, als ob er alles unter Kontrolle hätte. Wir wissen, dass es genau umgekehrt ist, und in diesem Sinne wäre eine gewisse Steifheit seiner Gebärden zu verstehen.

\section{Figuren}

Josef K. scheint mit seinem Vorbild aus dem Roman vieles gemeinsam zu haben. Wir lernen ihn hauptsächlich durch seine Beziehungen zu anderen Protagonisten der Bühnenbearbeitung kennen. Diese kann man in zwei Gruppen unterteilen: die einen treten bereits im Original auf, die anderen sind vom Regisseur frei erfunden. Paradoxerweise ist es eben die zweite Gruppe, die eine genauere Antwort auf die Frage gibt, wer Josef K. eigentlich sei. Bei der

3 Diese Szene erinnert an die Gestalt des alten Bendemann aus der Erzählung Das Urteil. 
Präsentation der meisten Personen aus der ersten Gruppe (Fräulein Bürstner, der Onkel, Advokat Huld, Leni, Block, der Priester) stützt sich Fiedor auf die Vorlage. Nur bei der Gestalt des Malers Titorelli ist eine Abweichung vom Original zu beobachten, etwa wenn ihm Homosexualität zugeschrieben wird. Aber der Regisseur hält sich dabei an die Romanstellen, die Max Brod aus seiner Proce $\beta$-Edition entfernte. Man kann also annehmen, dass die Darstellungsweise des Malers als eines Menschen mit homosexueller Neigung dem Entwurf Kafkas entspricht. Eine interessante und für die Aussage des Ganzen wesentliche Verfahrensweise ist der Ausbau von Figuren, die im Roman nur beiläufig erwähnt werden. Es handelt sich hier um die Mutter und die Krankenschwester, bei deren Darstellungsweise Fiedor sich ebenfalls auf in der Brod-Ausgabe unveröffentlichte Romanstellen stützt. Dementsprechend ist zu erfahren, dass die Mutter K.s sehr krank ist und dass er sie vernachlässigt. Die Krankenschwester gehört zu K.s Anklägern. Es werden auch die Gestalten des alten Paares und des Mannes mit rotem Bart im Fenster gegenüber ausgebaut, die in dem Stück als der Alte, die Alte und der Sohn auftreten. Dank dieser Figuren wird die Perspektive, von draußen ' eingeführt. Der Regisseur betrachtet also Josef K. anders, als es das in Polen geltende Stereotyp fordert, das zur Wahrnehmung K.s als eines kleinen Rädchens in der Maschinerie der Bürokratie führt, die ihn durch ihre Größe überwältigt. Der Held wird zu deren unschuldigem Opfer. Bei Fiedor sind eben die anderen Figuren die Opfer K.s, eines hochgestellten Bankbeamten, der nur an die eigene Karriere denkt. Bei der Verfolgung der eigenen Ziele, dem Erreichen weiterer Stufen in der Bankhierarchie, verfährt K. rücksichtslos. Das ist besonders deutlich in seiner Konfrontation mit den Figuren aus der Banksphäre zu sehen. Entweder nimmt er diese Menschen gar nicht wahr (die Sekretärin, den Fabrikanten), ist ihnen gegenüber aggressiv (die Putzfrau), skrupellos (Bankkunde, Arbeitsloser) oder schaut auf sie herab (Bankangestellter). Die Tatsache, dass die Figuren aus der Banksphäre keine Namen tragen und nur in ihren sozialen Rollen erscheinen, zeigt, dass die Korporation den Menschen seiner Individualität beraubt. Alle diese Figuren außer dem Fabrikanten - gehören zu der Gruppe der von Fiedor erfundenen Figuren, ebenso die Figuren der ersten Szene, die Fiedor mit der Bezeichnung ,alle“ versieht, die dann auch der Verhörszene beiwohnen, also die Globalisierungsgegner, die Krishna-Anhänger und die demonstrierenden Arbeiter. Alle diese Figuren haben eines gemeinsam: Sie gehören einer Welt an, die Josef K. nicht kennt. Er lebt in der Welt der gläsernen Bürohäuser, der modernen Wohnviertel, der angesagten Klubs (im Verhalten der Klubbesucher zeigt sich die Oberflächlichkeit der zwischenmenschlichen 
Beziehungen in dieser Welt besonders deutlich) und der teuren Restaurants. All diese Figuren sind auch K.s Ankläger in seinem Prozess, der als langsames Austreten aus seiner sozialen Isolation aufgefasst werden darf. Es ist eben der Prozess, der Josef K. dazu führt - oder besser gesagt dazu zwingt -, Kontakt mit Menschen aufzunehmen, mit denen er bis jetzt nichts zu tun hatte. Dieser Versuch, ihm die Augen zu öffnen über die Sinnlosigkeit eines durch das Erfolgsdenken geprägten Lebens, scheitert jedoch. Die Begegnung mit den oben erwähnten Figuren hat K. nichts gelehrt. Dafür wird er bestraft.

Die meisten Gerichtsrepräsentanten werden in dem Stück übergangen (der Richter, der Student, der Gerichtsdiener, der Prügler, der Kanzleidirektor) oder nur indirekt dargestellt (die Aufseher, die zwei Herren im Zylinder). Das Gericht, als K.s Gewissen aufgefasst, erfordert keine direkten Repräsentanten. Das Gericht ist in Josef K., und die anderen Personen erinnern ihn nur daran. Eine wichtige Bedeutung für die Aussage der gesamten Bühnenbearbeitung hat die Einführung der Engel, die K. in den Schlüsselmomenten seines Prozesses begleiten. Sie verkörpern den Prozess, wovon die achtzehnte Szene zeugt. Als der Prozess zu Ende geht und sie überflüssig zu sein scheinen, befestigt der Priester sie an der Decke, wo sie reglos, wie in Agonie, hängenbleiben. Dank dieser Figuren gewinnt die Bühnenbearbeitung einen metaphysischen Charakter, und die Interpretation des Romans durch Fiedor bleibt nicht einseitig. Die Vorstellung wird zu einem poetischen Bild.

\section{Die Inszenierung und ihre Rezeption}

Die Premiere der Bühnenbearbeitung von Franz Kafkas Der Proce $\beta$ in der Regie Marek Fiedors fand am 20. Februar 2004 im Teatr Polski in Poznań statt und wurde sowohl von der Theaterkritik als auch vom Publikum sehr positiv aufgenommen. Von der Beliebtheit des Stückes beim Publikum zeugt, von der hohen Besucherzahl abgesehen, eine Welle von Leserbriefen an die Redaktion der Gazeta Wyborcza. Diese Briefe waren eine Reaktion der empörten Zuschauer auf die einzige eindeutig negative Kritik, die EWA OBRĘBOWSKA-PIASECKA (2004) verfasst hatte. Die Kritikerin unterscheidet wobei sie sich der Richtigkeit ihrer Unterscheidung nicht sicher ist - drei Erzählebenen: eine psychologische, eine soziologische und eine metaphysische. Dabei ist sie der Meinung, dass diese drei Ebenen zu nichts geführt hätten. Die Dialoge hätten nicht überzeugt, die Schauspieler ,neben dem Text“" gesprochen. Zudem sei das Ganze nicht überzeugend genug gespielt worden. Auch wenn sie sich auf ausgeklügelte Ideen stützten, hätten die 
einzelnen Szenen den Zuschauer der szenischen Wahrheit, die zwischen der Bühne und dem Zuschauerraum entstehen sollte, nicht näher gebracht. Durchaus anderer Meinung war Katarzyna Tórz, die sich von der Vielfalt der Töne, der szenischen Poesie und dem perfekten Rhythmus der Fiedorschen Proce $\beta$-Vision begeistern ließ. Fiedor zeige, so TóRZ (2004), eine Welt, die mitten in der Krise stecke, und der Prozess Josef K.s sei beinahe als das Letzte Gericht zu sehen, das täglich in Millionen Köpfen stattfinde. Fiedor decke den Übermut und die Eitelkeit des Menschen auf, der glaube, die Welt ganz und gar zu verstehen. Den perfekten Rhythmus der Vorstellung lobt auch DOROTA JARZĄBEK (2004). Sie verweist auf die langsame Entwicklung der Handlung, die ihrer Ansicht nach den Rhythmus von K.s Prozess treffend widerspiegele. Auch habe Fiedor die Handlung aktualisiert, ohne dabei tendenziös zu werden, indem er K. als einen in der technokratischen Welt versunkenen Bankangestellten zeige. Die Aktualisierung von Fiedors Der Proce $\beta$ betont in seiner sehr wohlwollenden Kritik auch OLGIERD BŁAŻEWICZ (2004). Er vermisst zwar in der Vorstellung die Verankerung in der jüdischen Tradition, aber der aktuelle Kontext des Bühnengeschehens wirke überzeugend. Dabei hebt Błażewicz einige besonders gelungene Szenen hervor. Die Vorstellung Fiedors halte den Zuschauer dank der geheimnisvollen Atmosphäre in ihrem Bann. In einer weiteren Kritik bezeichnet BŁAŻEWICZ (2004a:14) die Bühnenfassung Fiedors als die erste Adaption von Kafkas Roman nach dem Fall der Berliner Mauer, was allerdings nicht der Wahrheit entspricht. Im Jahre 1993 präsentierte Ryszard Major eine Proceß-Bühnenfassung im Teatr Wybrzeże in Gdańsk, drei Jahre später folgte die Inszenierung von Henryk Baranowski im Teatr Rozmaitości in Warszawa. Einen sehr wichtigen Aspekt der Vorstellung bemerkt MAŁGORZATA KLAT (2004). Sie stellt fest, dass es Fiedor trotz der Vielfalt von Aktualisierungsverfahren gelungen sei, das Spektrum intellektueller Problematik, das Kafkas Text beinhaltet, zu bewahren. Derselben Meinung ist ANDRZEJ GóRNY (2004). Bei allen Bedenken, die die Darstellungsweise des Schicksals Josef K.s wecken könne, besitze das Ende der Bühnenfassung dennoch die aus dem Original bekannte Aussage. Eine Rückkehr zum Originaltext vermerkt auch BŁAŻEJ KUSZTELSKI (2004), der allerdings Fiedors Wendung nach der anfangs aktuell-realistischen Version als Versinken in einer unverständlichen, existentiellen Metaphorik wertet. Die rationale Satire werde zum unklaren, grotesken Mysterium. Zugleich weiß Kusztelski die nicht banale Regieführung, das Bühnenbild sowie die Schauspielkunst zu schätzen. Auf einen anderen Aspekt der Bühnenfassung kommen zwei weitere Kritiker zu sprechen. Sowohl JACEK SIERADZKI (2004) als auch MARTA MIELCAREK 
Eliza Szymańska

(2004) betonen die Befreiung vom Schema der Romanvorgabe, nach dem Josef K. ein finsterer armer Beamter und noch dazu Opfer des Gerichts sei. Beiden Kritikern gemeinsam ist eine gewisse hochtrabene Ausdrucksweise, mit der die günstigen Beurteilungen formuliert werden. Sieradzki zufolge gehöre die Frage nach einer transzendentalen Lebensdimension in der heutigen Welt zu den wichtigsten Aufgaben des Theaters. Nur selten sei dieses Anliegen so klug und deutlich realisiert worden wie in der Inszenierung Fiedors. MiELCAREK (2004:18) beginnt ihre Kritik mit den Worten: „Wenn Franz Kafka noch lebte, wäre er nach dem Ansehen der neuesten Bühnenfassung von seinem Der Prozeß im Teatr Polski in Poznań zufrieden.“ Im weiteren Teil ihrer Rezension versucht die Autorin diese Annahme zu belegen. Fiedors Bühnenfassung beurteilt sie als bahnbrechend, gut durchdacht und großartig zu unseren Zeiten passend. Fast alle Kritiker loben die Rolle Michał Kaletas (Josef K.). Auf positive Resonanz stoßen auch die Rollen von Roland Nowak (Maler Titorelli), Zina Kerste (Fräulein Bürstner), Katarzyna Węglicka (Putzfrau) und Edward Warzecha (Huld).

Der Proce $\beta$ von Marek Fiedor nahm an dem seit 1961 organisierten TheaterTreffen in Kalisz teil, das seit 1985 als Festival der Schauspielkunst gilt. Diese Tatsache wurde von zwei Rezensenten verzeichnet (AND 2004; BŁAŻEWICZ 2004b). Die Tatsache, dass Fiedors Der Proce $\beta$ an dem Festival teilnahm, sei - so BŁAŻEWICZ (2004c) - der beste Beweis dafür, dass nicht nur die in schauspielerischer, sondern auch in interpretatorischer Hinsicht besten Vorstellungen nach Kalisz eingeladen würden.

\section{Zusammenfassung}

Die Bühnenbearbeitung Fiedors von Kafkas Der Proceß reiht sich in die allgemeine polnische Kafka-Rezeption der letzten Jahre ein. Nachdem Josef $\mathrm{K}$. in den 80ern von den Regisseuren konsequent freigesprochen und als Opfer des totalitären Systems (lies: des kommunistischen Systems) dargestellt worden war, begann man in den 90ern wiederum, Kafka im Geiste der existentialistischen Philosophie zu interpretieren. Vor allem wurde auf das Absurde in Kafkas Werk verwiesen, weil es die Welt, in der wir zu leben haben, vortrefflich darstelle. Durch diese These wird die Aktualität von Kafkas Werk unterstrichen. Diese Aktualität wird noch deutlicher in den Vordergrund gestellt, wenn Kafkas Schaffen als Beschreibung eines von Angst erfüllten Lebens in der materialistischen Welt des Kapitalismus präsentiert wird (SOMMERFELD 2003). Den Bühnenfassungen von Kafkas Der Proceß in 
den 90er Jahren ist die existentialistische Auslegung anzumerken, allein schon dadurch, dass Josef $\mathrm{K}$. jedes Mal schuldig gesprochen wird. Und jedes Mal besteht die Schuld darin, dass er diese Schuld leugnet, und zwar in einer Welt, in der von Unschuld zu sprechen eine Zumutung ist. Josef K. negiert seine Schuld, weil er die Regeln, nach denen sich die Welt richtet, nicht kennt. Dafür wird er aufs härteste bestraft. Auch die Bühnenbearbeitung von Fiedor stellt die elementare Frage nach dem Wesen der Menschlichkeit, was uns erlaubt, sie in die Reihe der existentialistischen Auslegungen zu stellen. Eine mechanische, jeglichen Kontakts mit anderen Menschen entbehrende Lebensweise wird aufgezeigt, die zugleich als Schuld des Protagonisten verstanden wird. Besonders hervorzuheben ist auch der soziale Aspekt dieser Bühnenfassung von Kafkas Der Proceß. In diesem Zusammenhang bringen sich die beiden Bühnenbearbeitungen von Peter Weiss in Erinnerung (HEYDE 1997; ZIMMERMANN 1990). Es gibt aber einen entscheidenden Unterschied zwischen den Bearbeitungen von PeTER WeISS - Der Prozeß (1975), Der neue Prozess (1982) - und der Bühnenfassung von Fiedor. Bei Weiss war Josef K. ein kleines Rädchen in der Maschinerie der Bürokratie, die auf die Ausbeutung der Menschen eingestellt war. Der Protagonist wollte um jeden Preis ein Teil des Establishments sein, was ihn zur leichten Beute für andere machte, die ihn zu eigenen Zwecken zu nutzen wussten. Bei Fiedor ist K. bereits fast auf dem Gipfel seiner beruflichen Möglichkeiten angelangt. Er ist gerade derjenige, der die anderen zu eigenen Zwecken nutzt, da er sich dadurch eine Besserung seiner beruflichen Situation erhofft. Diese Darstellung Josef K.s als eines Teils des Establishments macht die Besonderheit der Bühnenbearbeitung Fiedors im Kontext der anderen polnischen Theaterfassungen von Der Proce $\beta$ aus (SZYMAŃSKA 2008). Eine weitere Besonderheit liegt in dem spezifischen Verständnis der Aktualität des Romans in der gegenwärtigen Welt. Die anderen Regisseure sahen die Aktualität Kafkas in der Universalität des Schicksals des Prokuristen Josef K. Fiedor erzeugt Aktualität zusätzlich durch die Einführung mehrerer Personen und Szenen, die man im Originaltext vergebens suchen würde, die aber die Handlung eindeutig am Anfang des 21. Jhd.s platzieren. Josef K. wird schuldig gesprochen. Seine Schuld liegt in der konformistischen Haltung einer Existenz, die nur nach Macht, Geld und beruflichen Erfolgen strebt. Dieser ständige Wettbewerb um eine bessere gesellschaftliche Position, der der Situation im postkommunistischen Polen entspricht, kann nach Meinung Fiedors nur übel enden. Zum ersten Mal wird auch die Sprache einem Aktualisierungsverfahren unterzogen. Fiedor geht mit dem Text des Originals sehr locker um. Jede der Aussagen im Stück ist jedoch in der einen oder anderen Form im 
Roman wiederzufinden, sei es als Gedanke des Protagonisten, sei es als Aussage einer anderen Person. Fiedor erlaubt sich also im Umgang mit Kafkas Text durchaus große Freiheiten, unterschlägt aber dennoch nichts von dessen wesentlicher Aussage. ${ }^{4}$

\section{Literatur}

AND (2004): Rusza aktorski festiwal. [Das Schauspielfest beginnt]. In: Gazeta Poznańska, 30.4.-3.5.2004:19.

BAyERDÖRFER, HANS-PETER (ed.) (2007): Vom Drama zum Theatertext? Tübingen.

BŁażewicz, Olgierd (2004): Rewizja Kafki. [Die Revision Kafkas]. In: Gtos Wielkopolski, 23.2.2004:11.

- (2004a): Rewizja „Procesu“. [Die Revision des Proceß]. In: Gtos Wielkopolski, 12.3.2004:14

- (2004b): Nad Prosna. [An der Prosna]. In: Gtos Wielkopolski, 23.4.2004:8.

- (2004c): Wygrali klasycy. [Die Klassiker haben gewonnen]. In: Gtos Wielkopolski, 11.5.2004:8

Canetti, Elias (2005): Der andere Prozeß. Kafkas Briefe an Felice. München.

FIEdor, MAReK (2004): Der Proceß nach Franz Kafka. Bühnenbearbeitung. Teatr Polski. Poznań [Theaterexemplar].

GÓRNY, ANDRZEJ (2004): Ktoś coś ode mnie chce. [Jemand will was von mir]. In: Gazeta Malarzy i Poetów 2:28-29.

Guderian-CZAPlińska, Ewa (2004): Rygor i sens. Rozmowa z Markiem Fiedorem. [Die Rigorosität und der Sinn. Ein Gespräch mit Marek Fiedor]. In: Didaskalia (Februar/April):34-36.

HEYDE, ANDREA (1997): Unterwerfung und Aufruhr. Franz Kafka im literarischen Werk von Peter Weiss. Berlin.

JARZĄBEK, DoRota (2004): Spór o wtasne istnienie. [Der Streit um das eigene Dasein]. In: Didaskalia (Februar/April):32-33.

KafKa, Franz (1997): Der Verschollene. Der Proceß. Das Schloß. Die Romane. Frankfurt (M.).

- (2000): Proces. Przeł. Bruno Schulz. [recte: Józefina Szelińska i Bruno Schulz]. Warszawa.

4 In ähnlicher Weise verfährt ein anderer bedeutender polnischer Regisseur der letzten Jahre - Piotr Cieplak - mit der Vorlage des Originals. In einem vor einiger Zeit gegebenen Interview gibt der Regisseur zu, im Original stets alles „,auf den Kopf zu stellen“, ohne dabei dessen wesentliche Aussage aus den Augen zu verlieren (USZYŃSKA 2004:6). 
Klat, Malgorzata (2004): Proces rozpoczęty. [Der Prozess hat begonnen]. In: Bezpłatny Tygodnik Poznański, 20.2.2004:16.

KusZTELSKI, BŁAŻEJ (2004): Oskarżony menedżer Józef K. [Der angeklagte Manager Josef K.]. In: Gazeta Poznańska, 23.2.2004:12.

Listy. [Briefe]. (2004): Gazeta Wyborcza (Poznań), 25.2.2004:21.

MielcareK, Marta (2004): Nowy proces. [Der neue Prozess]. In: Miasteczko Poznań 3:18-19.

OBRĘBOwSKA-PIASECKA, EwA (2004): Stowo honoru: nie rozumiem. [Ehrenwort: Ich verstehe nicht]. In: Gazeta Wyborcza (Poznań), 23.2.2004:11.

Polemiki. [Polemiken]. (2004): Gazeta Wyborcza (Poznań), 26.2.2004:22.

SIERADZKI, JACEK (2004): Józef K. jako człowiek sukcesu. [Josef K. als Erfolgsmensch]. In: Przekrój, 21.3.2004:38.

SOMMERFELD, BEATE (2003): Die Rezeption Franz Kafkas in der polnischen Literaturkritik. In: Convivium:119-164.

SZYMAŃSKA, ElizA (2008): Adaptacje sceniczne „Procesu“ Franza Kafki w Polsce. [Szenische Adaptationen des Proceß von Franz Kafka in Polen]. Wrocław.

TóRz, KatARzyna (2004): Sąd ostateczny. [Das Jüngste Gericht]. In: Teatr 1/2:24-25. UszyŃSKa, MARTa (2007): Rozmowa z Piotrem Cieplakiem. [Ein Gespräch mit Piotr Cieplak]. In: Didaskalia (Juni/August):4-6.

WeISs, Peter (1975): Der Prozeß. Stück in zwei Akten nach dem gleichnamigen Roman von Franz Kafka. In: Theater heute. Die deutsche Theaterzeitschrift 2:40-54.

- (1982): Der neue Prozess. Stück in drei Akten. Frankfurt (M.).

Zimmermann, Ulrike (1990): Die dramatische Bearbeitung von Kafkas „Prozeß“ durch Peter Weiss. Frankfurt (M.).

ŻUROwSKI, ANDRZEJ (2005): Kafka $w$ teatrze polskim. Rekonesans tematu badawczego. [Kafka im polnischen Theater. Erkundung eines Forschungsthemas]. In: KALINOWSKI, DANiEl (ed.): Twórczość Franza Kafki. Tożsamość kulturowa i literacka. Słupsk, 241-263. 